Revue internationale P.M.E.

Économie et gestion de la petite et moyenne entreprise

\title{
Qui sont les repreneurs potentiels d'entreprises? Proposition de typologie en fonction de l'état de santé de la firme
}

\author{
Mickael Geraudel, Annabelle Jaouen, Audrey Missonier et David Salvetat
}

Volume 22, numéro 3-4, 2009

URI : https://id.erudit.org/iderudit/044126ar

DOI : https://doi.org/10.7202/044126ar

Aller au sommaire du numéro

Éditeur(s)

Presses de l'Université du Québec

ISSN

0776-5436 (imprimé)

1918-9699 (numérique)

Découvrir la revue

Citer cet article

Geraudel, M., Jaouen, A., Missonier, A. \& Salvetat, D. (2009). Qui sont les repreneurs potentiels d'entreprises? Proposition de typologie en fonction de l'état de santé de la firme. Revue internationale P.M.E., 22(3-4), 13-30.

https://doi.org/10.7202/044126ar
Résumé de l'article

La question de recherche est la suivante : selon l'état de santé de la firme, quelles sont les caractéristiques des repreneurs potentiels ? Cet article vise à déterminer s'il est possible de caractériser les repreneurs potentiels et à établir qui sont les acteurs capables de reprendre certaines catégories d'entreprises. Les réponses apportées ici peuvent s'avérer particulièrement pertinentes pour les institutionnels en charge du développement économique de leurs territoires (CCI, incubateurs, pôles de compétitivité, etc.). Cette recherche repose sur une enquête menée en 2008 auprès de 44 porteurs de projet de reprise du Languedoc-Roussillon. Les résultats de la recherche, issus d'une analyse typologique, montrent que trois groupes de repreneurs potentiels se profilent : les prudents, les indifférents et les aventuriers. D’un point de vue managérial, cette recherche contribue à une meilleure connaissance des repreneurs potentiels et permet d'améliorer leur accompagnement dans leur démarche de reprise. 


\title{
Qui sont les repreneurs potentiels d'entreprises? Proposition de typologie en fonction de l'état de santé de la firme
}

\author{
Mickael GERAUDEL \\ Annabelle JAOUEN \\ Audrey MISSONIER \\ Groupe Sup de Co Montpellier \\ David SALVETAT \\ Groupe Sup de Co La Rochelle
}

\section{MOTS CLÉS}

Reprise - Repreneur - Intention - Typologie - Santé de l'entreprise

\begin{abstract}
LES AUTEURS
MiCKAEL GeRAUDEL est professeur assistant au Groupe Sup de Co Montpellier. II enseigne principalement la stratégie d'entreprise. Ses recherches portent sur les réseaux personnels des dirigeants de PME ainsi que sur les stratégies de coopétition développées par les grands acteurs industriels. Adresse: Groupe Sup de Co Montpellier, CEROM, 2300, avenue des Moulins, 34184 Montpellier, Cédex 4, France. Courriel : <m.geraudel@supco-montpellier.fr>.

ANNABELLE JAOUEN est professeure associée au Groupe Sup de Co Montpellier où elle enseigne l'entrepreneuriat et le management de projet. Ses domaines de recherche s'orientent sur la très petite entreprise (TPE), ses spécificités et ses comportements stratégiques. Elle travaille notamment sur les stratégies interorganisationelles de TPE: réseaux, alliances stratégiques, coopérations. Adresse: Groupe Sup de Co Montpellier, CEROM, 2300, avenue des Moulins, 34184 Montpellier, Cédex 4, France. Courriel :<a.jaouen@supco-montpellier.fr>.

AUDREY MISSONIER est professeure assistante au Groupe Sup de Co Montpellier. Elle enseigne la stratégie et l'entrepreneuriat. Ses recherches portent sur les opérations de rapprochements technologiques dans le secteur des TIC, la création d'innovation, la conduite du changement (changements structurels et culturels), les négociations et les modes de gouvernance. Elle est chef de projet depuis plusieurs années auprès de sociétés innovantes de la Netéconomie. Adresse: Groupe Sup de Co Montpellier, CEROM, 2300, avenue des Moulins, 34184 Montpellier, Cédex 4, France. Courriel : a.missonier@supco-montpellier.fr>.
\end{abstract}

DAVID SALVETAT est professeur associé au Groupe Sup de Co La Rochelle. II enseigne la stratégie d'entreprise et développe ses recherches au sein du laboratoire CEREGE sur des problématiques relatives aux stratégies de coopétition et sur la modélisation des pratiques d'intelligence économique. Adresse: Groupe Sup de Co La Rochelle, CEREGE, 102, rue des Coureilles - Les Minimes, 17024 La Rochelle, Cédex 1, France. Courriel : <salvetatd@ esc-larochelle.fr>. 


\title{
RÉSUMÉ
}

La question de recherche est la suivante: selon l'état de santé de la firme, quelles sont les caractéristiques des repreneurs potentiels ? Cet article vise à déterminer s'il est possible de caractériser les repreneurs potentiels et à établir qui sont les acteurs capables de reprendre certaines catégories d'entreprises. Les réponses apportées ici peuvent s'avérer particulièrement pertinentes pour les institutionnels en charge du développement économique de leurs territoires ( $\mathrm{CCl}$, incubateurs, pôles de compétitivité, etc.). Cette recherche repose sur une enquête menée en 2008 auprès de 44 porteurs de projet de reprise du Languedoc-Roussillon. Les résultats de la recherche, issus d'une analyse typologique, montrent que trois groupes de repreneurs potentiels se profilent: les prudents, les indifférents et les aventuriers. D'un point de vue managérial, cette recherche contribue à une meilleure connaissance des repreneurs potentiels et permet d'améliorer leur accompagnement dans leur démarche de reprise.

\begin{abstract}
The research question is the following one: according to the firm's health, what are the features of management buy-in realized by individuals? The goal of this article is double: to characterize individuals who want to buy-in companies, and to understand who are the people able to buy-in specific companies. The strength of this study is to propose contributions to help public and private actors who have in charge development of their territory (for example, chamber of commerce). The sample is composed of 44 individuals who have a project of management buy-in in the Languedoc Roussillon area. Thanks to a typological analysis, three types of entrepreneurs are identified: the adventurous, cautious and indifferent managers. On a managerial point of view, this research contributes to a better knowledge of potential manager buy-in in order to help them to develop their projects.
\end{abstract}

\section{RESUMEN}

La cuestión de investigación es la siguiente: ¿ según el estado de salud de la empresa, cuáles son las características de las personas que se hace cargo de la empresa en crisis potenciales? Este artículo tiene por objeto determinar si es posible caracterizar las personas que se hace cargo de la empresa en crisis potenciales, y a incluir que son los protagonistas capaces reanudar algunas categorías de empresas. Las respuestas establecidas aquí pueden resultar especialmente pertinentes para institucionales en carga del desarrollo económico de sus territorios (CCl, incubadoras, polos de competitividad, etc.). Esta investigación se basa en una investigación realizada en 2008 ante 44 portadores de proyecto de reanudación del Languedoc-Roussillon. Los resultados de la investigación, resultantes de un análisis tipológico, ponen de manifiesto que tres grupos de personas que se hace cargo de la empresa en crisis potenciales aparecen: los prudentes, los indiferentes y los aventureros. Desde un punto de vista de gestión, esta investigación contribuye a un mejor conocimiento de las personas que se hace cargo de la empresa en crisis potenciales con el fin de mejorar su acompañamiento en su planteamiento de reanudación. 


\section{ZUSAMMENFASSUNG}

Die Forschungsfrage lautet: Kann aufgrund des Zustandes eines Unternehmens auf die Eigenschaften eines potenziellen Übernehmers geschlossen werden? Ziel des vorliegenden Artikels ist die Diskussion der Frage, für welche Art von Unternehmen welche Nachfolger mit welchen Eigenschaften in Frage kommen. Die vorgeschlagenen Antworten sind besonders interessant für Institutionen, die in diesem Bereich tätig sind. Diese Forschung basiert auf einer Umfrage aus dem Jahre 2008 bei 44 Projektverantwortlichen für geplante Übernahmen im Languedoc-Roussillon. Die Cluster-Analyse ergab drei Gruppen von potenziellen Übernehmern: die Vorsichtigen, die Gleichgültigen und die Abenteurer. Aus betriebswirtschaftlicher Sicht trägt diese Forschung zu einem besseren Verständnis der potenziellen Übernehmer bei, um die Unterstützung im Übernahmeprozess zu verbessern.

\section{Introduction}

Au cours des 10 prochaines années, 550000 entreprises vont être cédées en France et une PME sur quatre va changer de dirigeant (Chabert, 2005). Si $88 \%$ des entreprises rachetées ne dépassent pas cinq salariés, la majeure partie d'entre elles (60\% en 2007, selon l'Insee) sont rachetées par des tiers, étrangers à la firme et sa culture. Ainsi, la reprise d'entreprise représente un enjeu économique et social considérable tant au regard du nombre d'entreprises concernées que des impacts organisationnels posés par le changement de dirigeant.

La reprise se définit comme le rachat d'une firme permettant de s'en rendre propriétaire: le repreneur seul ou avec sa famille, ou son (ses) allié(s), acquiert la majorité absolue du capital (Deschamps, 2002a). La reprise d'entreprise par des personnes physiques revêt quelques dimensions spécifiques dans le champ de l'entrepreneuriat (Deschamps, 2002b). À la différence de la transmission d'entreprise familiale (Cadieux, 2005), sa particularité réside dans la méconnaissance qu'a le dirigeant de l'entreprise dont il prend la direction. Si les recherches sur le sujet se développent de plus en plus, elles s'orientent autour de plusieurs dimensions: le «quoi reprendre», où l'on traite des questions d'évaluation des firmes, de ciblage et de potentiel de création de valeur de l'entreprise (Bruton, Oviatt et White, 1994; Barbot, 2002; Salvato, Lassini et Wiklund, 2007); le «comment reprendre», à savoir ex-ante les processus et étapes à suivre, mais aussi les dangers de la reprise (Vatteville, 1994); ou encore le «comment réussir la reprise», comme les problématiques relatives à l'intégration du néodirigeant et à sa stratégie (Haddadj et D'Andria, 1998; Picard et Thévenard-Puthod, 2004; Boussaguet, 2007). Enfin, un ensemble de travaux s'intéressent au «qui reprend», soit au repreneur et à son profil (Bienaymé,1981; Trigano et Lafougère, 1986; Siegel, 1989; Deschamps, 2002a). 
La présente recherche se positionne dans cette dernière catégorie, en s'intéressant aux repreneurs potentiels d'entreprise. L'objet de cette recherche est de caractériser les repreneurs potentiels en fonction de l'état de santé de la firme ciblée. En effet, parmi les thématiques porteuses, tant d'un point de vue théorique que managérial, la question de l'état de santé de la firme rachetée reste peu étudiée. Peut-on caractériser les repreneurs potentiels selon l'état de santé de la firme recherchée (saine, en difficulté ou en redressement judiciaire)? Pour répondre à cette question, cette recherche se fonde sur une enquête menée depuis 2008 auprès de 44 repreneurs potentiels du Languedoc-Roussillon ayant manifesté officiellement leur souhait de racheter une entreprise.

Les résultats, issus d'une analyse typologique, montrent que trois groupes de repreneurs potentiels apparaissent: les prudents, qui ne cherchent que des entreprises en bonne santé et refusent d'envisager le rachat d'entreprise en difficulté; les aventuriers, preneurs de risques et recherchant en priorité des entreprises en difficulté (c'est-à-dire déficitaire depuis plus de deux exercices comptables) ou en redressement judiciaire; enfin, les indifférents, qui ne manifestent pas d'intérêt particulier pour l'une ou l'autre des catégories. Chaque groupe présente des caractéristiques propres qui seront précisées plus avant. Ainsi, après avoir fait état de la littérature sur les types de repreneurs et les intentions de reprise, l'article apporte un éclairage sur le protocole de recherche, pour ensuite présenter la typologie de repreneurs potentiels. Celle-ci est analysée et discutée et les auteurs mettent finalement ces éléments en perspective et développent les intérêts managériaux de la recherche.

\section{Cadre conceptuel}

\subsection{Les types de repreneurs d'entreprise}

La communauté scientifique s'accorde à considérer le repreneur comme une catégorie d'entrepreneur à part entière (Verstraete, 1999), en tant qu'individu à l'origine d'une organisation (non réduite à la seule création d'entreprise), ou de la détection-construction-exploitation d'une occasion d'affaires, de la création de valeur ou d'une innovation (Verstraete et Fayolle, 2005). Les travaux visant à améliorer la connaissance du repreneur d'entreprise sont en constant développement et plusieurs typologies sont proposées par la communauté scientifique mais aussi par des éditorialistes de presse économique, chacun 
se centrant sur un aspect différent: les modifications apportées à l'entreprise (Bienaymé, 1981), la stratégie (Siegel, 1989), la formation (Daigne, 1995), le lien avec la cible (Deschamps, 2002a) ou le type de contraintes subies (Abdesselam, Bonnet et Le Pape, 2004). Le tableau 1 de la page suivante en propose un résumé. Il caractérise les repreneurs en fonction de diverses dimensions en montrant que le passage à l'acte de reprise semble être le résultat d'une combinaison des quatre éléments suivants:

- Les motivations du repreneur (goût d'entreprendre, souhait d'indépendance, idée nouvelle);

- Un ensemble de contraintes (accès aux ressources financières, informationnelles ou techniques). Abdesselam, Bonnet et Le Pape (2004) notent que la disposition à l'action entrepreneuriale ou repreneuriale est expliquée non seulement par des caractéristiques psychologiques (le sentiment de pouvoir agir sur son devenir) mais aussi par des trajectoires de vie (caractéristiques sociales touchant à la fois à des évènements de rupture professionnelles, personnelles ou culturelles, etc.);

- L'environnement du repreneur, notamment l'existence d'un entourage entrepreneurial et l'accès aux ressources nécessaires à la mise en œuvre du projet (par exemple, les technopoles). Abdesselam, Bonnet et Le Pape (2004) soulignent également que cet environnement est en partie déterminé par la localisation géographique du repreneur;

- L'expérience préalable du repreneur, désireux de se lancer dans la possession et la gestion d'une nouvelle entreprise (MacMillan, 1986; Westhead et Wright, 1998; Westhead, Ucbasaran et Wright, 2005). Politis (2008) montre notamment que l'expérience acquise et l'apprentissage qu'elle permet de réaliser peut améliorer la performance des entreprises et leur probabilité de survie.

Cependant, l'aptitude à prendre des risques ou, au contraire, l'aversion pour le risque, n'apparaît pas comme un facteur déterminant dans ces typologies. Si l'aversion pour le risque est une variable explicative du comportement entrepreneurial (Kilhstrom et Laffont, 1979; Van Praag et Cramer, 2001; Kan et Tsai, 2006), la question, bien que pertinente, est peu posée dans le domaine de la reprise d'entreprise. 
TABLEAU 1

Les typologies de repreneurs

\begin{tabular}{|c|c|c|}
\hline Auteur(s) & Typologie & Point de mire \\
\hline $\begin{array}{l}\text { Shapero } \\
(1975)\end{array}$ & $\begin{array}{l}\text { Repreneur push } \\
\text { Repreneur pull }\end{array}$ & $\begin{array}{l}\text { Motivations } \\
\text { à la reprise. }\end{array}$ \\
\hline $\begin{array}{l}\text { Bienaymé } \\
\text { (1981) }\end{array}$ & $\begin{array}{l}\text { Repreneur remplaçant une direction défaillante. } \\
\text { Repreneur restructurant l'entreprise par une } \\
\text { modification substantielle de son portefeuille d'activités. } \\
\text { Repreneur démantelant l'entreprise. }\end{array}$ & $\begin{array}{l}\text { Modification } \\
\text { infligée à } \\
\text { la substance } \\
\text { de l'entreprise. }\end{array}$ \\
\hline $\begin{array}{l}\text { Trigano et } \\
\text { Lafougère } \\
(1986)\end{array}$ & $\begin{array}{l}\text { Héritier } \\
\text { Salarié de l'entreprise. } \\
\text { Tiers }\end{array}$ & Nature \\
\hline
\end{tabular}

Astreints ou repreneurs « réactifs» réagissant à une situation plus que ne la provoquant (crainte du chômage, obligation de succéder au père...).

Siegel Entrepreneurs ou repreneurs «proactifs» se (1989) caractérisant par l'esprit d'entreprise.

Stratégie

Créateurs reprenant pour mettre en œuvre une idée nouvelle d'output.

Repreneurs «bidon» ou «chasseurs de primes».

\begin{tabular}{|c|c|c|}
\hline $\begin{array}{l}\text { Daigne } \\
(1995)\end{array}$ & $\begin{array}{l}\text { Commercial } \\
\text { Financier } \\
\text { Technicien }\end{array}$ & Formation \\
\hline $\begin{array}{l}\text { L'Entreprise } \\
\text { (1995) }\end{array}$ & $\begin{array}{l}\text { Repreneur souhaitant maîtriser seul les règles du jeu. } \\
\text { Repreneur souhaitant éviter le «syndrome du } \\
\text { couperet» (cadre de } 45 \text { ans qui a peur d'être licencié). } \\
\text { Repreneur défendant une équipe et un produit. } \\
\text { Repreneur réalisant une plus-value. } \\
\text { Repreneur licencié. }\end{array}$ & $\begin{array}{l}\text { Motivations } \\
\text { de l'individu }\end{array}$ \\
\hline $\begin{array}{l}\text { L'Usine } \\
\text { Nouvelle } \\
\text { (1995) }\end{array}$ & $\begin{array}{l}\text { Investisseur riche } \\
\text { Gestionnaire } \\
\text { Professionnel } \\
\text { Chômeur } \\
\text { Touriste } \\
\text { Collaborateur }\end{array}$ & $\begin{array}{l}\text { Expérience } \\
\text { professionnelle }\end{array}$ \\
\hline $\begin{array}{l}\text { Deschamps } \\
\text { (2002a) }\end{array}$ & $\begin{array}{l}\text { Ancien salarié. } \\
\text { Ancien partenaire commercial. } \\
\text { Membre de la famille du cédant. } \\
\text { Actionnaire minoritaire. } \\
\text { Repreneur sans lien avec la cible. }\end{array}$ & Lien repreneur-cible \\
\hline $\begin{array}{l}\text { Abdesselam, } \\
\text { Bonnet } \\
\text { et Le Pape } \\
(2004)\end{array}$ & $\begin{array}{l}\text { Repreneur non contraint. } \\
\text { Repreneur contraint mais inséré. } \\
\text { Repreneur non informé. } \\
\text { Repreneur non expérimenté et isolé. }\end{array}$ & $\begin{array}{l}\text { Nature des } \\
\text { contraintes subies } \\
\text { et évolution de } \\
\text { la création } \\
\text { d'emplois générés }\end{array}$ \\
\hline
\end{tabular}

Source: Adapté et complété de Deschamps (2002a). 


\subsection{Risque et intention de reprise}

Des recherches récentes portant sur la perception du risque et l'intention entrepreneuriale montrent que le risque peut être perçu soit comme une menace, soit comme une opportunité (Fayolle, Barbosa et Kickul, 2008) et détermine les choix de création-reprise. Ainsi, la plus ou moins grande propension à prendre des risques ou à percevoir une situation comme risquée pourrait déterminer la propension à rechercher une entreprise saine ou en difficulté. Outre le risque financier du rachat d'entreprise, les risques d'ordre organisationnel et culturel sont majeurs, notamment dans le rachat d'entreprise en difficulté (Deschamps et Paturel, 2005): difficulté de remettre les salariés au travail, risque de comportement de sabotage, sous-évaluation du passif, non-réalisation de plus-values latentes, sous-estimation du «poids » de l'ancien dirigeant, etc. Daigne (1995) classe ainsi les risques liés à la reprise en quatre catégories: risques sociaux (conflits de culture, rejet de la part des cadres, démission des hommes clés), risques managériaux (divergences dans les systèmes de management, trop grande centralisation, incompréhension interne et externe), risques financiers (problèmes de trésorerie, sous-estimation de la planification financière, défaillance du système d'information) et risques stratégiques (mauvais diagnostic d'acquisition, réactions de la concurrence, crainte des fournisseurs ou des clients, perte de parts de marché). Dickson et Giglierano (1986), repris ensuite par Fayolle, Barbosa et Kickul (2008), décomposent le risque entrepreneurial en deux dimensions: le risque d'échec et le risque de manquer une opportunité.

Ces risques, bien que souvent sous-estimés (Deschamps et Paturel, 2005), sont généralement perçus par les repreneurs potentiels. Aussi, l'aversion pour le risque semble-t-elle jouer un rôle majeur dans la phase d'intention de reprise, notamment lorsqu'on s'interroge sur le choix de reprendre une entreprise saine ou en difficulté. L'intention de reprendre une entreprise occupe une place centrale dans le processus de décision (Emin, 2003): l'intention reflète en effet la vision entrepreneuriale et a pour effet les actions concrètes qu'elle a impulsées. Varraut (1999) décrit «l'intention» comme émergeant d'un «décalage entre la représentation actuelle d'un avenir (vision stratégique) et la représentation actuelle du présent», déclenchant ainsi «une tension dans l'esprit du dirigeant, tension qui peut être à l'origine de l'émergence de l'intention d'atteindre cet état futur (jugé avantageux par rapport à la représentation de la situation présente). La représentation de l'avenir se muerait alors en objet désiré et induirait une intention stratégique» (p. 44). Si l'intention constitue le moteur, le déclencheur de l'action de reprendre, Ajzen (1991) indique que la propension à prendre des risques influe sur cette intention. 
Le risque de défaillance de l'entreprise en difficulté est plus grand que chez une entreprise saine. Par conséquent, le repreneur d'une entreprise en difficulté doit supporter un risque plus élevé compte tenu de l'état de santé de cette entreprise. Nous pouvons ainsi penser que le repreneur désireux de racheter une entreprise en difficulté aura une aversion pour le risque moins grande qu'un repreneur recherchant une entreprise saine. Alors que cette dimension semble pertinente pour l'analyse des repreneurs potentiels, la notion de risque n'apparaît pas dans les typologies de repreneurs relevées. Pourtant, cette prise en compte peut apporter des éléments de compréhension des intentions de reprise, notamment quant à l'état de santé de la firme recherchée. Cette recherche, qui vise à caractériser les repreneurs potentiels en fonction de l'état de santé de la cible, met notamment en exergue cette propension.

\section{Méthode de recherche}

La recherche a été menée auprès de repreneurs potentiels ${ }^{1}$ de la région Languedoc-Roussillon. La région Languedoc-Roussillon se dote en effet d'une politique ambitieuse en faveur de la transmission d'entreprises: elle permet de préserver les firmes existantes, leur savoir-faire et leurs emplois à travers un réseau fédéré d'acteurs chargés de détecter et d'accompagner les projets de transmission-reprise, d'une part, et des outils financiers régionaux dédiés à la transmission-reprise, d'autre part (11,5 millions d'euros par an). De plus, la nouvelle politique régionale a récemment créé un dispositif d'aide à la reprise, à la transmission et à la création: le PACTE (Programme d'appui à la création et à la transmission d'entreprise). Ce programme a un double objectif: financer les projets de transmission et création (prêt, garantie et subvention) et former le repreneur au métier de chef d'entreprise.

Un questionnaire a été envoyé à des repreneurs dont le projet de reprise est connu ou abouti, ainsi qu'à des associations qui proposent un accompagnement dans la reprise d'entreprise (par exemple, la Soridec, les chambres consulaires, etc.). Quarante-quatre questionnaires se sont révélés être exploitables. Cette enquête s'applique à tous secteurs et à toutes tailles d'entreprises. Les 11 variables construites et leurs items sont présentés dans le tableau 2.

1. Sont appelés « repreneurs potentiels» les individus qui se sont manifestés auprès des institutions régionales et départementales du Languedoc-Roussillon, en se disant intéressés par un projet de reprise d'entreprise. 


\section{TABLEAU 2}

\section{Variables et items}

\begin{tabular}{|c|c|c|}
\hline Variables & Items & Codage \\
\hline \multirow{3}{*}{$\begin{array}{l}\text { 1. La situation actuelle } \\
\text { du repreneur }\end{array}$} & Au chômage & $\overline{\left(\mathrm{SIT} \_\mathrm{CHO}\right)}$ \\
\hline & Ingénieur ou cadre & (SIT_ING) \\
\hline & Dirigeant ou gérant & (SIT_DIR) \\
\hline \multirow{3}{*}{$\begin{array}{l}\text { 2. Le mode de } \\
\text { financement } \\
\text { de la reprise }\end{array}$} & Par fonds propres & (FI_FP) \\
\hline & $\begin{array}{l}\text { Par l'aide financière de la famille, } \\
\text { des amis }\end{array}$ & (FI_AF) \\
\hline & $\begin{array}{l}\text { Par un endettement extérieur tel } \\
\text { que les banques ou les investisseurs. }\end{array}$ & (FI_EE) \\
\hline \multirow{4}{*}{$\begin{array}{l}\text { 3. L'expérience } \\
\text { professionnelle } \\
\text { précédente }\end{array}$} & Indépendant & (JOB_INDEP) \\
\hline & TPE & (JOB TPE) \\
\hline & $\mathrm{PME} / \mathrm{PMI}$ & (JOB_PME) \\
\hline & Grande entreprise & (JOB_GE) \\
\hline \multirow{7}{*}{$\begin{array}{l}\text { 4. Le secteur recherché } \\
\text { par le repreneur }\end{array}$} & $\begin{array}{l}\text { Secteur similaire dans lequel } \\
\text { le repreneur a déjà travaillé }\end{array}$ & (MEME_SECT) \\
\hline & Secteur primaire & (PRIM_SECT) \\
\hline & Secteur industriel & (INDUS_SECT) \\
\hline & Secteur commercial & (COM_SECT) \\
\hline & Secteur du BTP & (BTP_SECT) \\
\hline & Secteur des transports & (TRANS_SECT) \\
\hline & Secteur des services & (SERV_SECT) \\
\hline \multirow{5}{*}{$\begin{array}{l}\text { 5. La taille de } \\
\text { l'entreprise recherchée }\end{array}$} & Aucun salarié & (RECH_AUCUN) \\
\hline & 0 à 4 salariés & $\left(\mathrm{RECH} \_4\right)$ \\
\hline & 5 à 9 salariés & (RECH_9) \\
\hline & 10 à 19 salariés & (RECH_19) \\
\hline & 20 à 40 salariés & ( $\left.\mathrm{RECH} \_40\right)$ \\
\hline \multirow{3}{*}{$\begin{array}{l}\text { 6. L'état de santé de } \\
\text { l'entreprise recherchée } \\
\text { par le repreneur }\end{array}$} & Entreprise en bonne santé & (RECH_SANTE), \\
\hline & Entreprise en difficulté & (RECH_DIF) \\
\hline & Entreprise en redressement & (RECH_REDRES) \\
\hline \multirow{4}{*}{$\begin{array}{l}\text { 7. Le lieu de l'entreprise } \\
\text { recherchée }\end{array}$} & Près du lieu de résidence & (LIEU_RES) \\
\hline & Dans un rayon de $300 \mathrm{~km}$ & (LIEU_300) \\
\hline & N'importe où en France & (LIEU_FRANCE) \\
\hline & À l'étranger & (LIEU_ETRAN) \\
\hline $\begin{array}{l}\text { 8. La correspondance } \\
\text { formation / activité } \\
\text { professionnelle }\end{array}$ & Formation/activité & (CORRESP_PRO) \\
\hline \multirow{5}{*}{$\begin{array}{l}\text { 9. La formation } \\
\text { du repreneur } \\
\text { (Variables de contrôle) }\end{array}$} & CAP & (FORM_CAP) \\
\hline & BEP & (FORM_BEP) \\
\hline & BAC & (FORM_BAC) \\
\hline & BTS & (FORM_BTS) \\
\hline & Diplôme supérieur & (FORM_PLUSBAC) \\
\hline \multirow{4}{*}{$\begin{array}{l}\text { 10. L'âge du repreneur } \\
\text { (Variables de contrôle) }\end{array}$} & 20 à 30 ans & $\left(\mathrm{AG} \_30\right)$ \\
\hline & 30 à 40 ans & $\left(A G \_40\right)$ \\
\hline & 40 à 50 ans & $\left(A G \_50\right)$ \\
\hline & Au-delà de 50 ans & (AG_PLUS50) \\
\hline \multirow{2}{*}{$\begin{array}{l}\text { 11. Le genre du repreneur } \\
\text { (Variables de contrôle) }\end{array}$} & Homme & (SEXE_HOM) \\
\hline & Femme & (SEXE_FEM) \\
\hline
\end{tabular}


Il est également nécessaire d'apporter quelques précisions terminologiques quant à la notion d'entreprise en difficulté. Selon Ferry-Maccario et al. (2006), le concept d'entreprise en difficulté est relativement récent. Le Code de commerce, article L. 631-1, note qu'une entreprise doit être considérée comme étant en difficulté dès que le débiteur «justifie des difficultés qu'il n'est pas en mesure de surmonter, de nature à le conduire à la cessation de paiements ». La loi définit la cessation de paiements comme «une entreprise qui ne peut plus faire face à son passif exigible avec son actif disponible» (ibidem). Il est donc à noter que, normalement, le traitement des entreprises en difficulté s'applique à celles qui ne sont pas encore en cessation de paiements. Nous avons ajouté le critère d'entreprise réalisant des pertes depuis plus de deux exercices comptables.

L'objectif de la recherche est de connaître quelles sont les caractéristiques des repreneurs potentiels. Cela suppose deux phases:

- segmenter l'échantillon en fonction de variables pertinentes pour connaître les différents types de repreneurs potentiels;

- caractériser les individus des sous-groupes créés.

Dans un premier temps, nous avons mené une analyse typologique hiérarchique et non hiérarchique grâce aux dendrogrammes, nuées dynamiques et k-means (Bouroche et Saporta, 1980; Evrard, Pras et Roux, 2003). Ces méthodes ont permis de fixer un nombre de catégories a priori et a posteriori. Compte tenu du caractère exploratoire de la recherche, une stratégie heuristique a consisté à comparer les différentes configurations proposées et à ne retenir que celle jugée comme la plus satisfaisante (Punj et Steward, 1983; Evrard, Pras et Roux, 2003). Ainsi, nous avons conservé la typologie qui repose sur trois groupes. Nous avons ainsi pu déterminer trois profils de repreneurs potentiels: les prudents, les indifférents et les aventuriers. Cette typologie a été réalisée à partir de la variable centrale de notre questionnaire, soit celle relative à l'état de santé de l'entreprise recherchée (RECH_SANTE, RECH_DIF et RECH_REDRES). Cette variable est considérée dans notre recherche comme une proxi de l'aversion pour les risques et notamment pour les risques financiers.

Dans un second temps, nous avons construit une matrice des corrélations afin d'étudier les liaisons entre les items. Cette analyse descriptive nous a permis de connaître les forces et pouvoirs de liaison entre items. L'objectif de cette deuxième étape est de comprendre quels sont les individus qui composent les catégories et, grâce aux Lambdas de Wilks, les préférences individuelles correspondant à une intention de reprise. 


\section{Résultats et discussion}

Tout d'abord, les résultats permettent de caractériser le répondant type de notre questionnaire (personnes physiques ayant une intention déclarée de reprise): c'est un homme (il n'y a que $5 \%$ de femmes), âgé de 40 à 50 ans, qui a fait des études post-bac. Il favorise le financement par fonds propres et a, en général, déjà travaillé dans des PME ou dans des grandes entreprises. Il souhaite trouver une entreprise plutôt proche de son lieu de résidence. Précisons ici qu'il s'agit de la description générale de l'échantillon. Présentons maintenant les profils identifiés.

L'analyse typologique a mis en évidence la coexistence de trois types de repreneurs que nous avons pu caractériser ainsi: les prudents, les indifférents et les aventuriers. Cette typologie a été réalisée en fonction de leur aversion pour le risque au regard de l'état de santé de l'entreprise recherchée (ou, en d'autres termes, de leur préférence à racheter une entreprise saine ou en difficulté). Cette taxonomie est issue de l'analyse des k-means (Evrard, Pras et Roux, 2003). Puis, une fois les profils établis, la description de ces groupes a été affinée par l'analyse des relations interitems pour chaque profil de repreneur.

\subsection{Les prudents}

Les prudents préfèrent racheter des entreprises saines. Ils ne s'orientent pas vers le rachat d'entreprises en difficulté ou en redressement qui présente trop de risques pour eux. Les prudents sont des individus actuellement au chômage et qui recherchent un financement par endettement extérieur, pour cibler des entreprises industrielles ou de services.

L'étude des corrélations interitems a fait apparaître cinq corrélations significatives:

- Le souhait de reprendre une TPE est positivement corrélé avec l'expérience acquise en tant que dirigeant $(0,652 * *)^{2}$. A contrario, cela est négativement corrélé pour les individus au chômage $\left(-0,435^{*}\right)$;

- Quand l'individu est faiblement diplômé, il préférera s'orienter vers la reprise de TPE $(0,522 * *)$ alors qu'un individu plus diplômé n'est pas intéressé par la très petite structure $(-0,531 * *)$;

- Pour une entreprise de taille moyenne, le repreneur potentiel est prêt à se déplacer n'importe où en France $(0,692 * *)$;

2. Significativité ${ }^{*}<5 \%$; $* *<1 \%$; $* * * 0,1 \%$. 
- Lorsque l'individu est au chômage, il est prêt à reprendre une entreprise qui n'est pas proche de son lieu de résidence $\left(-0,525^{* *}\right)$ et même une entreprise éloignée de plus de $300 \mathrm{~km}\left(0,406^{*}\right)$;

- Dans le cadre d'une reconversion professionnelle, quand les repreneurs recherchent une entreprise dans le secteur du BTP,ils n'estiment pas nécessaire d'avoir une expérience dans ce secteur $\left(-0,408^{*}\right)$.

Comme Deschamps (2002a) l'indique au sujet des caractéristiques du «repreneur sans lien avec la cible», les prudents préfèrent reprendre une TPE saine. Ils sont peu enclins à prendre un risque entrepreneurial du fait de leur situation, la plupart étant au chômage. Cependant, ils sont prêts à se déplacer n'importe où en France pour reprendre une entreprise de taille moyenne. Cette situation de chômage va de pair avec une nécessité de financement du projet de reprise par endettement extérieur. En d'autres termes, ils ne peuvent procéder par autofinancement et doivent donc bien évaluer leur projet avant de s'engager. En effet, le fait d'être chômeur rend plus difficile la prise de risque puisque l'on pourrait penser qu'un échec de la reprise les conduirait dans une situation de grande précarité.

\subsection{Les aventuriers}

À la différence, les aventuriers ont tendance à favoriser la recherche d'entreprises en difficulté et/ou en redressement. Ils ont le goût du risque et aiment relever les défis entrepreneuriaux. Les aventuriers sont prêts à prendre certains risques sous certaines conditions: ils recherchent en priorité des petites entreprises (de 0 à 20 salariés), dans un secteur d'activité qu'ils connaissent bien. Ils sont prêts à se déplacer dans un rayon de $300 \mathrm{~km}$. Ils font appel aux banques et aux financements extérieurs pour financer leur projet.

La corrélation interitems n'a pas donné de résultats significatifs dans cette catégorie. L'aventurier doit donc tirer sa particularité d'autres éléments que nous n'avons pas mesurés. Par exemple, l'aventurier a peut-être une forte confiance en lui qui l'incite à rechercher des entreprises en difficulté que les conditions dans l'environnement soient favorables ou non.

\subsection{Les indifférents}

La catégorie des indifférents correspond aux repreneurs qui n'ont pas de préférence quant à la santé de l'entreprise reprise. Les indifférents ont exercé des fonctions de dirigeant, privilégient les financements par fonds propres et recherchent à reprendre des entreprises dans tous types de secteurs (industrie, services, etc.). 
Dans cette catégorie, trois corrélations interitems sont apparues significatives:

- Il existe une corrélation entre le fait que l'individu ait travaillé dans une TPE et qu'il recherche une entreprise dans le même secteur $\left(0,677^{*}\right)$. En outre, le fait d'avoir travaillé en TPE l'incite à rechercher une entreprise entre 0 et 4 salariés $\left(0,673^{*}\right)$.

- La corrélation met en évidence que les femmes repreneurs de la catégorie indifférents recherchent plutôt de très petites structures $\left(0,680^{*}\right)$, au contraire des hommes $(-0,589 *)$.

- Dans le cadre d'une reconversion professionnelle, les repreneurs recherchent principalement une entreprise du secteur commercial même s'ils n'ont pas d'expérience dans ce secteur $\left(-0,640^{*}\right)$.

Les repreneurs indifférents ne se distinguent pas particulièrement par leur aversion pour les risques. Cela pourrait s'expliquer par le fait qu'ils disposent de ressources financières plus importantes que les autres individus de l'échantillon. Ainsi, leur choix de financer le rachat par fonds propres pourrait expliquer que l'aversion pour le risque ne soit pas un élément privilégié dans leur choix de reprise d'entreprise ${ }^{3}$.

Plus généralement, notre contribution corrobore les résultats de Ronstadt (1988), MacMillan (1986) ainsi que Westhead et Wright (1998) qui insistent sur l'importance de l'expérience entrepreneuriale dans le repreneuriat. Celle-ci apparaît comme prépondérante dans le cas des repreneurs prudents et indifférents, expérimentés et ayant déjà dirigé des TPE ou travaillé en indépendants. En outre, il existe plusieurs différences en termes de genre. Les femmes appartenant à la catégorie indifférents recherchent plutôt de très petites structures, ce qui est le contraire des hommes. Cela rejoint les résultats de Cliff (1998) pour qui les entreprises tenues par des femmes tendent à être plus petites que celles tenues par des hommes. De plus, une autre différence distingue les hommes et les femmes: le mode de financement. Les hommes de la catégorie prudents favorisent le financement par fonds propres plutôt que par l'aide familiale alors qu'il n'apparaît aucune préférence pour les femmes en termes de mode de financement.

3. Voir les travaux de Evans et Jovanovic (1989) dans un contexte de création d'entreprise. 


\section{Conclusion}

La reprise d'entreprise reste un champ académique peu exploré. En effet, si l'on considère la récente reconnaissance de l'entrepreneuriat comme une discipline des sciences de gestion, il est aisé de comprendre que le champ du repreneuriat reste encore à baliser. Pourtant, le repreneur en tant que tel se distingue fortement de l'entrepreneur. Sa logique d'action n'est pas la même et son projet est également différent. Comparer l'intention de création à l'intention de reprise d'entreprise conduit inéluctablement à se tromper dans la compréhension des processus de décision des repreneurs potentiels. Ainsi, le processus d'intention de reprise doit être un champ de recherche à part entière. La mise en perspective de trois profils de repreneurs potentiels est une première étape dans la structuration des recherches sur l'intention de reprise.

Sur le plan théorique, notre recherche vise à élargir les connaissances sur les repreneurs d'entreprise. Pour ce faire, nous avons introduit le concept d'aversion pour le risque en tant qu'élément incontournable pour comprendre la propension des porteurs de projet à opter pour le choix d'une entreprise en fonction de l'état de santé de celle-ci. Ce concept fortement développé dans les disciplines voisines comme la psychologie ou le marketing est encore peu utilisé dans les recherches sur le repreneuriat alors qu'il est déjà central dans les recherches sur la création d'entreprise (Evans et Jovanovic, 1989; Kan et Tsai, 2006; Fayolle, Barbosa et Kickul, 2008).

D'un point de vue managérial, le principal intérêt de la recherche réside dans le fait qu'elle interroge les caractéristiques sociodémographiques du repreneur potentiel. En d'autres termes, elle vise à répondre à la question: quel repreneur serait potentiellement adapté à quelle entreprise, notamment en fonction de l'état de santé de cette firme? De nombreux acteurs institutionnels souhaitent en effet identifier en amont les caractéristiques des repreneurs afin de mieux les accompagner dans leur démarche en les orientant vers des projets adaptés à leur profil. Par exemple, dans le cas de reprise de TPE en grande difficulté ou en redressement, les aventuriers sont la catégorie de repreneurs potentiels qui semble la plus pertinente pour ce cas de figure. En effet, ils sont prêts à recourir aux banques pour financer ce projet à condition que cela soit une TPE d'un secteur qu'ils connaissent bien. À cette logique de reprise en fonction d'une connaissance et d'une expertise dans un secteur s'oppose une logique de polyvalence: celle des indifférents. Ces repreneurs potentiels qui n'ont pas de préférence quant à l'état de santé de l'entreprise recherchent principalement une entreprise dans le secteur commercial même s'ils n'ont pas d'expérience dans ce secteur. Autrement dit, il n'y a pas de recherche de proximité sectorielle. Cela s'explique par deux éléments: la grande polyvalence de ces repreneurs potentiels qui sont pour la plupart des anciens dirigeants d'entreprises et leur potentiel financier. 
Comme toute recherche, cette étude présente quelques limites. La première réside dans la méthode adoptée, la typologie étant en effet un exercice statistique empreint de subjectivité. Même si les trois classes retenues sont celles qui nous sont apparues comme la solution la plus satisfaisante, cette dernière demeure discutable. Cependant, la double analyse typologique hiérarchique et non hiérarchique favorise la recherche de la solution optimale. Ainsi, la segmentation retenue semble être la plus pertinente. La seconde limite repose sur la non-prise en compte de certains aspects économiques liés à la reprise. Le différentiel de prix entre une entreprise saine et une entreprise en difficulté ne serait-il pas un critère affectant largement la décision de reprise des acteurs? Plus que l'aspect coût qui reste relatif au niveau de revenu des repreneurs, il serait pertinent d'intégrer une variable mesurant le niveau de revenu de l'individu pour pouvoir comparer directement les repreneurs entre eux sur cet aspect. En effet, selon le montant de leurs patrimoines économique et financier, les acteurs seront plus ou moins enclins à investir dans la reprise d'entreprise saine ou en difficulté. Un acteur ayant un fort patrimoine économique et financier verra son investissement comme une action dans un portefeuille global de risques alors que pour un chômeur, la reprise peut représenter le projet de sa vie. Toutefois, pour Kan et Tsai (2006), il ne semble pas toujours évident que ces deux dimensions aient des retombées distinctes en termes de création d'entreprise ou aient un effet l'une sur l'autre. Selon Kan et Tsai (2006), les différents travaux arrivent à des résultats contradictoires à ce sujet. Il semble alors prometteur de conjuguer l'analyse des deux dimensions dans une prochaine recherche.

Un autre axe de réflexion consisterait à envisager que d'autres traits de personnalité puissent avoir un pouvoir explicatif fort sur l'intention de reprise. Par exemple, le self-efficacy ou «efficacité personnelle perçue» est le sentiment de l'individu relatif à sa propre efficacité en termes d'actions. Se perçoit-il comme efficace? Les travaux de Sherrod et al. (1977) montrent qu'une personne qui pense que son environnement est contrôlable se perçoit comme plus efficace. La relation de l'individu à son environnement va donc déterminer la perception qu'il possède au sujet de son efficacité. Dans le même ordre d'idées, le locus of control (Rotter, 1966), ou «lieu de contrôle », renvoie à l'idée qu'un individu pense être en mesure de diriger ou non son avenir. Les individus ayant un «lieu de contrôle interne» croient que leurs propres actions déterminent les récompenses qu'ils obtiennent, leurs succès, leur futur; par exemple, la réussite de leur éventuelle reprise d'entreprise. En revanche, les individus ayant un «lieu de contrôle externe» pensent que les succès qu'ils peuvent obtenir ne dépendent pas d'eux-mêmes et sont hors de leur contrôle; par exemple, la réussite de la reprise dépendra de la durée de la crise économique actuelle. Dans cette perspective, un repreneur «prudent» pourrait reprendre une entreprise en difficulté s'il pense qu'il 
a les atouts pour mener à bien son projet. Explorer cette dimension de la personnalité du potentiel repreneur semble être un élément important pour de futures recherches.

Une seconde voie de recherche serait d'identifier les projets qui débouchent réellement sur des reprises d'entreprises. Autrement dit, comment passe-t-on de l'intention à l'action? Qui sont les repreneurs qui se lancent réellement dans l'aventure repreneuriale et qui dépassent le stade du projet? À l'aide d'une étude longitudinale, la démarche consisterait à mettre en perspective les profils de repreneurs qui réussissent dans leur projet de reprise. Ainsi, les acteurs économiques institutionnels (CCI, incubateurs, pôle de compétitivité, etc.) pourraient établir un processus d'aide à la reprise basé sur les facteurs clés de succès de la reprise d'entreprise.

\section{Bibliographie}

Abdesselam, R., J. BonNeT et N. Le PAPE (2004), «Les contraintes de la création et de la reprise d'entreprises: une application aux régions françaises », Cahiers d'économie et de sociologie rurales, $\mathrm{n}^{\circ}$ 72, p. 33-68.

AJZEN, I. (1991), «The theory of planned behavior», Organizational Behavior and Human Decision Processes, vol. 50, p. 179-211.

BArbot, M.-C. (2002), «Évaluation des PME défaillantes dans le cadre d'un plan de cession », Revue française de gestion, $\mathrm{n}^{\circ}$ 140, septembre-octobre, p. 121-143.

BIENAYMÉ, A. (1981), «Les repreneurs», Revue française de comptabilité, no 116, p. 332-343.

Bouroche, J.M. et G. SAPORTA (1980), L'analyse des données, Paris, Presses universitaires de France, coll. «Que sais-je?».

BOUSSAGUET, S. (2007), «Réussir son entrée dans l'entreprise: le processus de socialisation organisationnelle du repreneur», Économies et sociétés, vol.41, n ${ }^{\circ}$, p. 145-163.

BRUton, G.D., B. OviatT et M.A. White (1994), «Performance of acquisitions of distressed firms», Academy of Management Journal, vol. 37, p. 972-989.

CADIEUX, L. (2005), «La succession dans les PME familiales: vers une compréhension plus spécifique de la phase du désengagement », Journal of Small Business and Entrepreneurship, vol. 18, $\mathrm{n}^{\circ}$ 3, p. 343-356.

CHABERT, R. (2005), Transmission d'entreprise: optimiser la prise de relais, Paris, Pearson Education France.

CLIFF, J.E. (1998), «Does one size fit all? Exploring the relationship between attitudes towards growth; gender and business size», Journal of Business Venturing, vol. 13, p. 523-542.

DAIGne, J.-F. (1995), Ré-ingenierie et reprise d'entreprise, Paris, Economica, coll. «Gestion». 
Deschamps, B. (2002a), «Proposition d'une typologie des profils de repreneurs d'entreprises», Actes du II ${ }^{e}$ Congrès de l'Académie de l'entrepreneuriat, Bordeaux, 17-18 avril.

DESCHAMPS, B. (2002b), «Les spécificités du processus repreneurial », Revue française de gestion, $\mathrm{n}^{\circ} 138$, p. 175-188.

DESCHAMPS, B. et R. PATUREL (2005), Reprendre une entreprise, saine ou en difficulté, $2^{\mathrm{e}}$ édition, Paris, Dunod.

DiCKSON, P.R. et J.J. GigLIERANO (1986), «Missing the boat and sinking the boat: a conceptual model of entrepreneurial risk», Journal of Marketing, vol. 50, $\mathrm{n}^{\circ} 3$, p. 58-70.

EMIN, S. (2003), L'intention de créer une entreprise des chercheurs publics: le cas français, Thèse de doctorat, Université de Toulon-Var.

L'ENTREPRISE (1995), «Cadres, comment devenir patrons? », n 116, mai, p. 40-53.

L'Usine Nouvelle (1995), «Reprendre une PMI aujourd'hui», n 2488, 2 février, p. 42-47.

EVANS, D.S. et B. JOVANOVIC (1989), «An estimated model of entrepreneurial choice under liquidity constraints », Journal of Political Economy, vol. 97, nº 4, p. 808-827.

Evrard, Y., B. Pras et E. RouX (2003), Market: études et recherches en marketing, $3^{\mathrm{e}}$ édition, Paris, Dunod.

FAYOLLE, A., S.D. BARBOSA et J. KiCKUL (2008), «Une nouvelle approche du risque en création d'entreprise », Revue française de gestion, vol. 185, p. 141-159.

Ferry-Maccario, N., J. Kleinheisterkamp, F. Lenglart, K. Medjad et N. StOlOWy (2006), Gestion juridique de l'entreprise, Paris, Pearson Education France, $368 \mathrm{p}$.

HADDADJ, S. et A. D’ANDRIA (1998), «Transmissions internes et transmissions externes dans les PME françaises: existe-t-il des différences de changements stratégiques et d'orientations stratégiques? », Revue internationale PME, vol.11, $\mathrm{n}^{\circ} 4$, p. 45-65.

KAN, K. et W.D. TSAI (2006), «Entrepreneurship and risk aversion», Small Business Economics, vol. 26, p. 465-474.

KiHLSTROM, R.R. et J.J. LAFFONT (1979), «A general equilibrium entrepreneurial theory of new firm formation based on risk aversion», Journal of Political Economy, vol. 87, p. 304-316.

MACMILLAN, I.C. (1986), «To really learn about entrepreneurship, let's study habitual entrepreneurs », Journal of Business Venturing, vol. 1, nº 3, p. 241-243.

PICARD, C. et C. THÉVENARD-PUTHOD (2004), «La reprise d'entreprise artisanale: spécificités du processus et conditions de réussite », Revue internationale PME, vol $17, \mathrm{n}^{\circ} 2$, p. 93-121.

Politis, D. (2008), «Does prior start-up experience matter for entrepreneurs' learning? A comparison between novice and habitual entrepreneurs », Journal of Small Business and Enterprise Development, vol. 15, n 3, p. 472-489. 
PUnJ, G. et D.-W. STEWARD (1983), «Cluster analysis in marketing research: review and suggestions for application», Journal of Marketing Research, vol. 20, p. $134-148$.

RONSTADT, R. (1988), «The corridor principle», Journal of Business Venturing, vol.3, p. 31-40.

ROTTER, J.B. (1966), «Generalized expectancies for internal control of reinforcement», Psychological Monographs, vol. 80, n 1, p. 609-633.

SALVATO, C., U. LASSINI et J. WiKLUND (2007), «Dynamics of external growth in SMEs : a process model of acquisitions capabilities emergence », Schmalenbach Business Review, vol. 59, n 3, p. 282-305.

Shapero, A. (1975), «The displaced, uncomfortable entrepreneur», Psychology Today, novembre, vol. 9, n 6 , p. 83-88.

SHERROD, D.R., J.N. HAGE, P.L. HALPERN et B.S. MOORE (1977), «Effects of personal causation and perceived control on response to an aversive environment: the more control, the better», Journal of Experimental Social Psychology, vol. 13, no 1 , p. 14-27.

SIEGEL, D. (1989), Contribution en vue d'une démarche stratégique de la reprise d'entreprise par des particuliers: le cas alsacien et franc-comtois, Thèse de doctorat en Sciences de gestion, Université de Grenoble II.

TrigAnO, G. et S. LAFOUgÈRE (1986), Transmission et reprise d'entreprise, Paris, Berger Levrault, coll. «Entreprise».

VAN PRAAG, C.M. et J.S. CRAMER (2001), «The roots of entrepreneurship and labour demand: individual ability and low risk aversion », Economica, vol. 68, n 269, p. 45-62.

VARRAUT, N. (1999), «De la vision à l'intention stratégique: une application au dirigeant-propriétaire de PME», Économies et Sociétés, Sciences de gestion, vol. 26-27, nos 6-7, p. 146-166.

VATTEVILlE, E. (1994), «Le risque successoral», Revue française de gestion, vol. 98, p. 29-49.

Verstraete, T. (1999), Entrepreneuriat, Connaître l'entrepreneur, comprendre ses actes, Paris, L'Harmattan, coll. «Économie et innovation».

Verstraete, T. et A. FAyOlle (2005), «Paradigmes et entrepreneuriat», Revue de l'entrepreneuriat, vol. 4, $\mathrm{n}^{\circ}$ 1, p. 34-52.

WESTHEAD, P. et M.WRIGHT (1998), «Novice, portfolio, and serial founders: are they different? », Journal of Business Venturing, vol. 13, p. 173-204.

WestheAd, P., D. UCBASARAN et M. Wright (2005), «Decisions, actions, and performance: do novice, serial, portfolio entrepreneurs differ?», Journal of Small Business Management, vol. 43, p. 393-417. 